\title{
Mucinous cystadenocarcinoma of the pancreas with anaplastic carcinoma: A case report and review of the literature
}

\author{
MASAYA MUNEKAGE ${ }^{1}$, TAKUHIRO KOHSAKI ${ }^{2}$, SUNAO UEMURA ${ }^{1}$, \\ HIROYUKI KITAGAWA $^{1}$, TSUTOMU NAMIKAWA ${ }^{1}$ and KAZUHIRO HANAZAKI ${ }^{1}$ \\ ${ }^{1}$ Department of Surgery, Kochi Medical School, Kochi University; ${ }^{2}$ Department of Gastroenterology and Hepatology, \\ Kochi Medical School, Kochi University, Nankoku, Kochi 783-8505, Japan
}

Received August 27, 2015; Accepted December 21, 2015

DOI: $10.3892 / \mathrm{mco} .2016 .743$

\begin{abstract}
Few reports of mucinous cystic neoplasm (MCN) in association with anaplastic carcinoma exist. The present study reported an unusual case of a 25-year-old female exhibiting large pancreatic MCN with anaplastic carcinoma. Notably, the patient was a Jehovah's Witness and therefore refused any blood transfusions. Preoperative diagnosis was invasive pancreatic MCN measuring $12.5 \mathrm{~cm}$ with ascites. Distal pancreatectomy was performed in combination with splenectomy and partial resection of the transverse colon. Intraoperative estimated blood loss was $400 \mathrm{ml}$, therefore a blood transfusion was not required. The patient had an uneventful postoperative course. The pathological diagnosis was mucinous cystadenocarcinoma of the pancreas with anaplastic carcinoma. Although the patient underwent postoperative adjuvant chemotherapy with gemcitabine and oral fluoropyrimidine (S-1), recurrence with peritoneal dissemination was detected 20 months following surgery and the patient succumbed to the recurrence 32 months following surgery. To the best of our knowledge, this is the first case report of MCN with anaplastic carcinoma of the pancreas in a Jehovah's Witness patient undergoing pancreatic surgery.
\end{abstract}

\section{Introduction}

Mucinous cystic neoplasm (MCN) of the pancreas is characterized by mucin-producing columnar epithelium and dense ovarian-type stroma. The prognosis for MCNs treated with complete surgical resection without any additional treatment is excellent, particularly for the non-invasive type $(1,2)$. By contrast, anaplastic carcinoma of the pancreas has a poorer prognosis compared with poorly differentiated ductal adeno-

Correspondence to: Dr Tsutomu Namikawa or Dr Masaya Munekage, Department of Surgery, Kochi Medical School, Kochi University, Kohasu, Oko-cho, Nankoku, Kochi 783-8505, Japan

E-mail: tsutomun@kochi-u.ac.jp

E-mail: m-munekage@kochi-u.ac.jp

Key words: pancreas, mucinous cystadenocracinoma, anaplastic carcinoma, distal pancretectomy, Jehovah's Witnesses carcinoma of the pancreas (3-7). Anaplastic carcinoma of the pancreas is a rare pancreatic tumor of epithelial origin, frequently presenting various morphologies, which include pleomorphic epithelial cells and relatively mononuclear spindle cells $(3,4)$. MCN in association with anaplastic carcinoma has rarely been reported to date (3-7).

The present case study reported an unusual case of a 25-year-old female exhibiting a large pancreatic MCN with anaplastic carcinoma. In addition, since the patient was a Jehovah's Witness who refused blood transfusion, intraoperative blood loss had to be minimized $(8,9)$. To the best of our knowledge, this is the first case report of MCN with anaplastic carcinoma of the pancreas in a Jehovah's Witness patient undergoing pancreatic surgery.

\section{Case report}

A 25-year-old female diagnosed with a large abdominal cystic tumor in a referring hospital was admitted to Kochi Medical School Hospital (Kochi, Japan). Although the past medical history of the patient was not notable, the patient was a Jehovah's Witness and therefore refused blood transfusions. Physical examination suggested the presence of an elastic hard mass in the left upper quadrant of the abdomen. Laboratory tests on admission revealed anemia (hemoglobin, $10.8 \mathrm{~g} / \mathrm{dl}$; hematocrit, $33.8 \%$ ) and a marginal elevation in the white blood cell count $(9,400 / \mu \mathrm{l})$. Although cancer antigen (CA)19-9 levels were normal, CA125 and detection of a pancreatic cancer-associated antigen-2 levels were elevated (178 and $710 \mathrm{U} / \mathrm{ml}$, respectively; normal $<35 \mathrm{U} / \mathrm{ml}$ each). Abdominal computed tomography (CT) revealed a large complex cystic and solid mass measuring $10 \times 12.5 \mathrm{~cm}$ in the body and tail of the pancreas with a thick capsule (Fig. 1) and ascites in the pouch of Douglas. Endoscopic retrograde cholangiopancreatography revealed disruption of the major pancreatic duct, the duct of Wirsung, in the body of the pancreas (Fig. 2).

A laparotomy was subsequently performed. No evidence of distant or systemic metastases were observed. The present study investigated cytology for ascites during the operation, which was negative for neoplastic cells. En bloc distal pancreatectomy with lymphadenectomy, splenectomy and partial resection of the transverse colon was performed (Fig. 3). The cut surface of the cystic mass exhibited a smooth external 


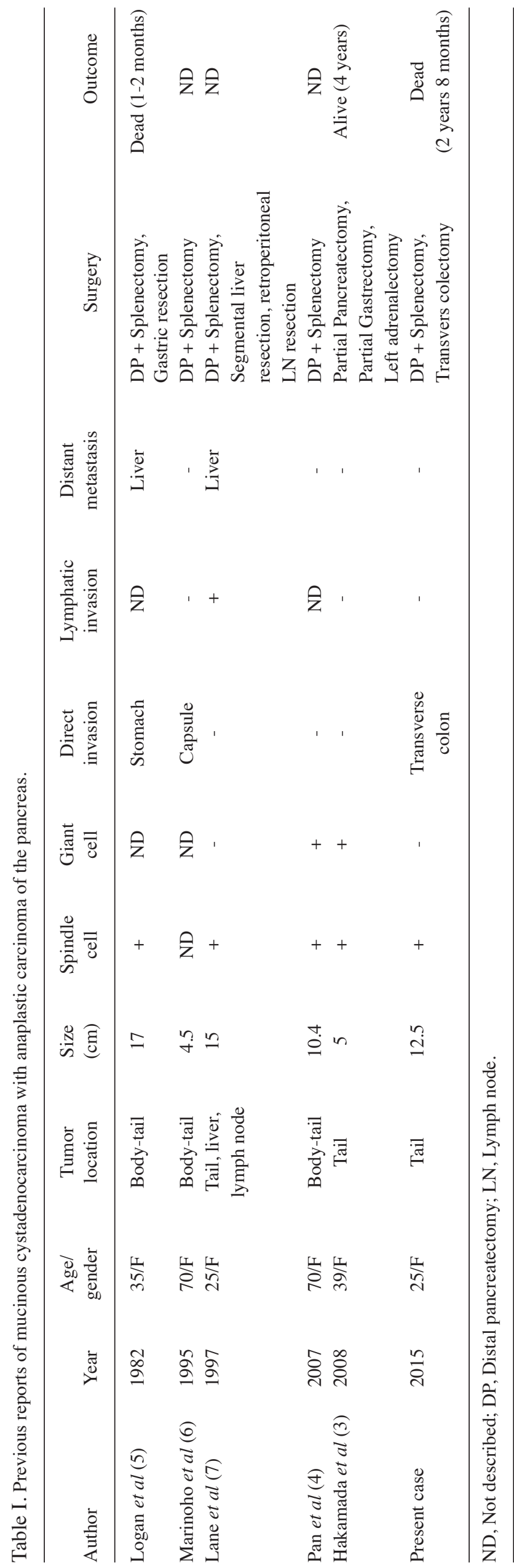

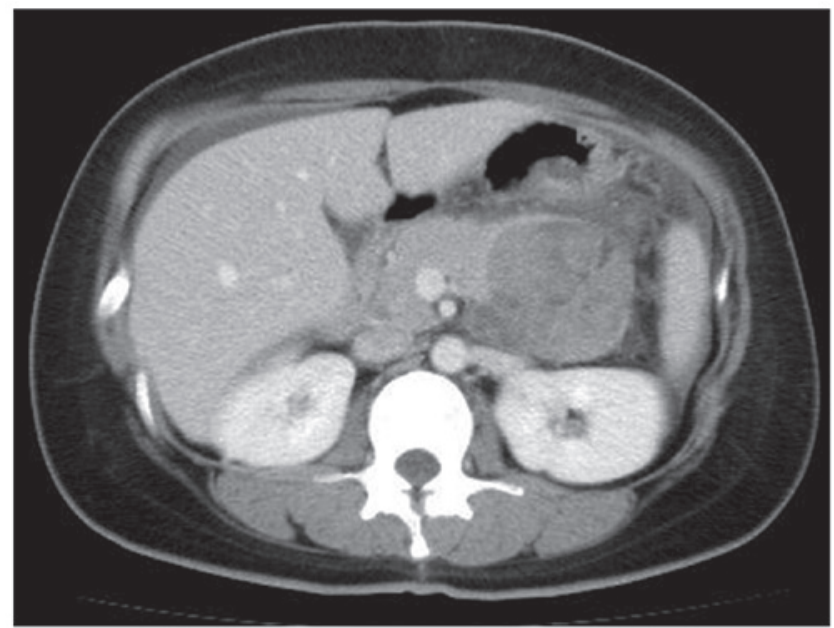

Figure 1. Computed tomography revealed a large complex cystic and solid mass in the body and tail of the pancreas.

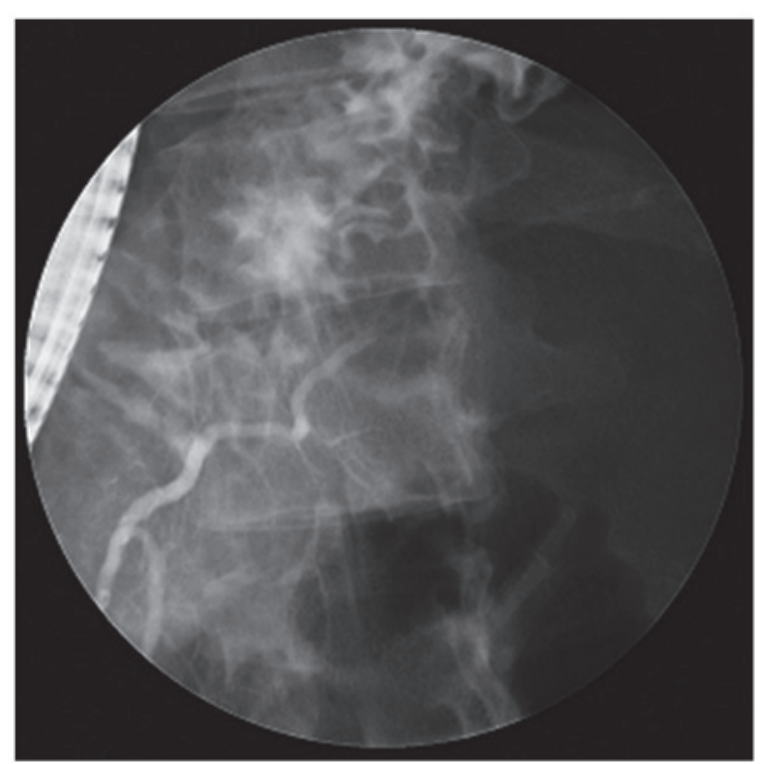

Figure 2. Endoscopic retrograde cholangiopancreatography revealed disruption of the duct of Wirsung in the body of the pancreas.

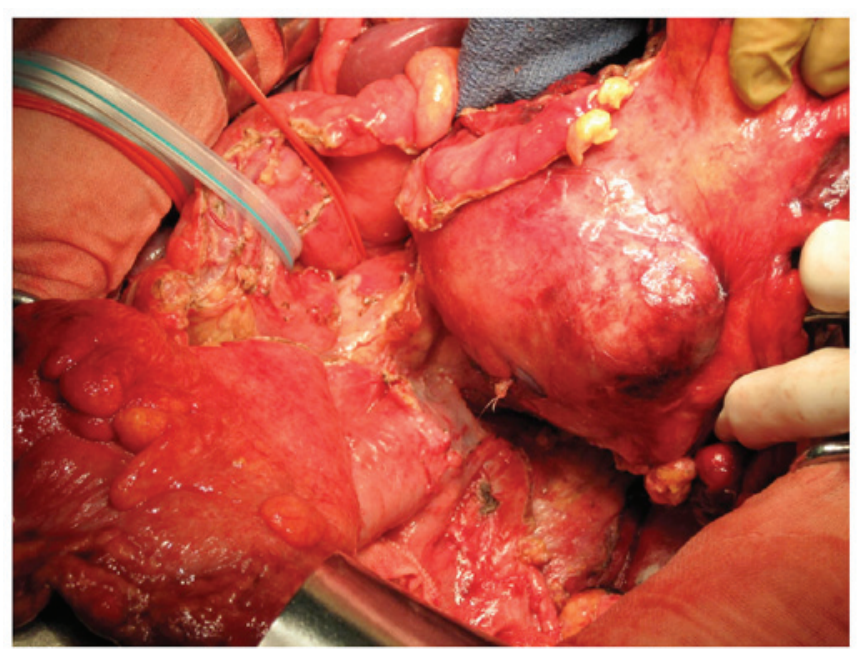

Figure 3. Distal pancreatectomy combined with splenectomy and partial resection of the transverse colon was performed. 


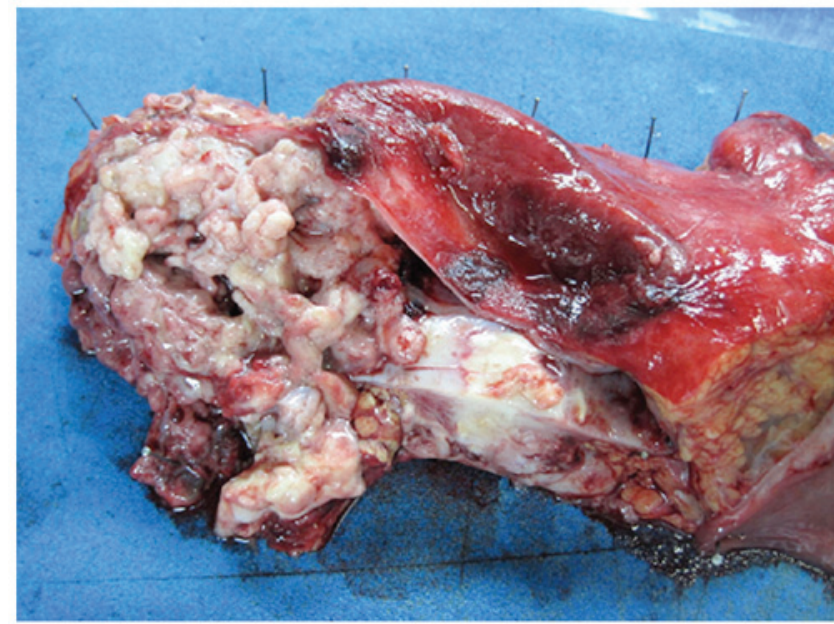

Figure 4. The cut surface of the cystic mass exhibited a smooth external surface. The mass was filled with mucinous fluid with a solid component.

surface, and it was filled with mucinous fluid and a solid component (Fig. 4). Histopathological analysis revealed MCN compatible with ovarian-type stromal tissue (Fig. 5A). Immunohistochemical analysis revealed positive staining for progesterone receptor in stromal cell nuclei (Fig. 5B). In addition, anaplastic carcinoma was suggested since the spindle and pleomorphic tumor cells stained positive for cytokeratin AE1/AE3 and vimentin in the solid area of the tumor (Fig. 5C). The pathological diagnosis was mucinous cystadenocarcinoma of the pancreas with anaplastic carcinoma, which penetrated the serosa without lymphovenous and retroperitoneal invasion. The mass was pT3N0M0 Stage III, according to the General Rules for the Study of Pancreatic Cancer edited by the Japan Pancreas Society (10).

The postoperative course was uneventful and the patient was discharged $\sim 1$ month following surgery. Following discharge, the patient underwent postoperative chemotherapy consisting of gemcitabine $\left(1,000 \mathrm{mg} / \mathrm{m}^{2}\right)+\mathrm{TS}-1\left(100 \mathrm{mg} / \mathrm{m}^{2}\right)$. During chemotherapy, the patient was performing well, enjoying life, and was able to undertake light sports and trips without any trouble. Unfortunately, recurrence (peritoneal dissemination) was detected by follow-up CT 20 months following surgery. Following detection of the recurrence, the patient was treated with chemoradiotherapy and subsequently palliative care at the hospital in her home town. The patient succumbed to pancreatic cancer 32 months following the surgery.

\section{Discussion}

Reports of MCN with anaplastic carcinoma of the pancreas are rare. To the best of our knowledge, only six cases (3-7) have been reported to date, including the present case (Table I). No previous patients have been a Jehovah's Witness. The prognosis for MCN is reasonably good, with a postoperative 5-year survival rate of almost $100 \%$ for adenoma, non-invasive and in situ carcinoma, and 30-60\% for invasive carcinoma (2). A multi-institute study in Japan reported a 5-year survival rate for MCN following resection of $100 \%$ in the case of adenoma to minimally invasive carcinoma, and $37.5 \%$ in the case of invasive carcinoma (1). By contrast, anaplastic carcinoma of
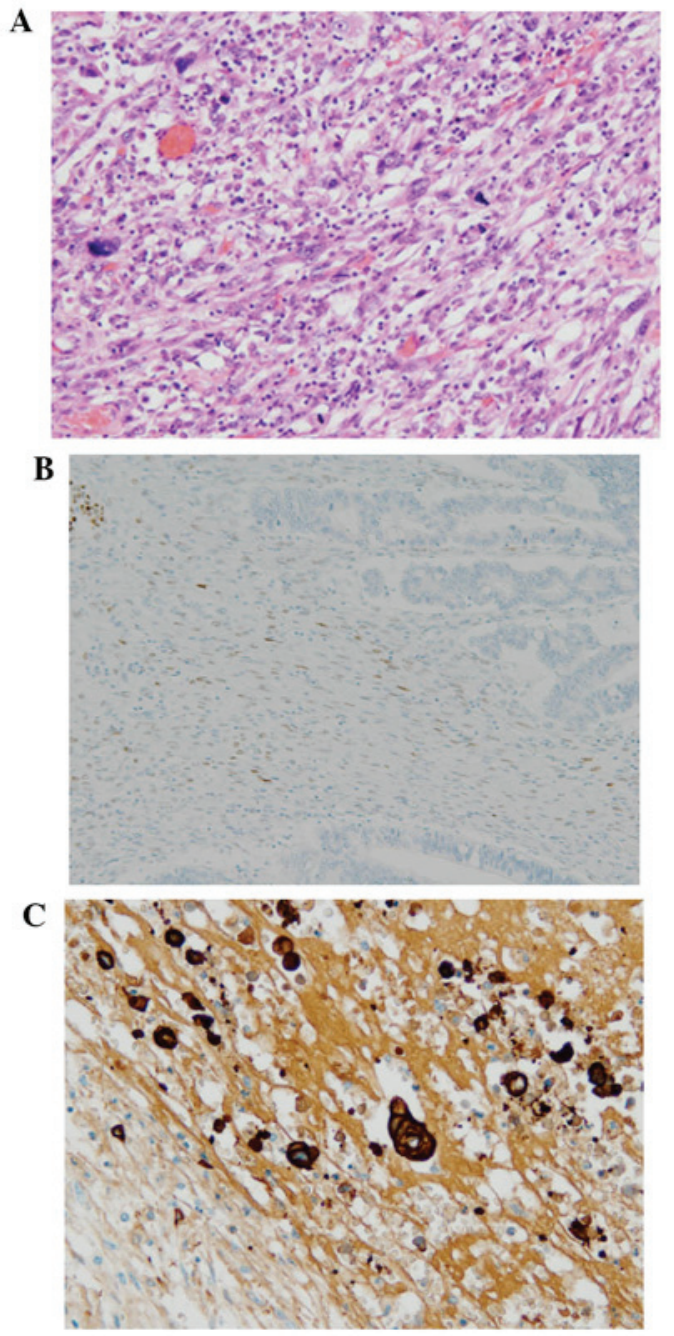

Figure 5. (A) Histopathological analysis revealed mucinous cystic neoplasm compatible with ovarian-type stromal tissue. (B) Immunohistochemistry revealed positive staining for progesterone and estrogen receptors in stromal cell nuclei. (C) Spindle and pleomorphic tumor cells were positive for immunohistochemical staining for both cytokeratin AE1/AE3 and vimentin in the solid area of the tumor, compatible with anaplastic carcinoma.

the pancreas is a rare, aggressive tumor. It accounts for 2-7\% of all pancreatic cancer types and has a male preponderance (2).

It is difficult to determine any differences in imaging modalities between MCN and MCN with anaplastic carcinoma, since this diagnosis was confirmed by the immunohistochemical features of epithelial components pathologically $(3,4)$. However, it is important to recognize this distinct entity, which is an aggressive neoplasm with a poorer prognosis compared with poorly differentiated ductal adenocarcinoma of the pancreas (4). The prognosis of pancreatic anaplastic carcinoma is extremely poor since, in most cases, the tumor not only has areas of adenocarcinoma, but also mesenchymal components, including spindle-shaped cells and osteoclastoid giant cells, reminiscent of sarcomatous differentiation $(3,4)$. Notably, no previous reports demonstrates any patients surviving $>5$ years following surgery, including the present case (Table I). Until now, the longest reported survival following surgery was 4 years (3).

Konstantinidis et al (9) reported a median estimated blood loss of $400 \mathrm{ml}$ for 10 pancreatic 
resections (six pancreaticoduodenectomy, four distal pancreatectomy/splenectomy; range 250-1,800 ml). In the present case, the estimated blood loss was $400 \mathrm{ml}$, which is comparable with previous reports. Generally, if Jehovah's Witness patients undergoing pancreatectomy experience massive blood loss, they are administered erythropoietin and iron hydroxide sucrose complex. Fortunately, the present patient did not require treatment for anemia since no change in postoperative hemoglobin and hematocrit was observed compared with the preoperative levels. Previously, the Liga Sure ${ }^{\mathrm{TM}}$ (COVIDIEN) vessel-sealing system was used to reduce intraoperative blood loss during proximal and/or distal pancreatectomy (11), and this system was used in the present patient. Regardless, in the case of Jehovah's Witness patients undergoing pancreatic resection, surgical outcome can be enhanced by good interdepartmental cooperation, careful patient selection and appropriate preoperative management.

A previous report suggested that lymph node metastasis occurred in $58 \%$ of surgical patients with invasive MCN (2). Therefore, pancreatic resection and standard lymphadenectomy (D1 or D2) against invasive MCN of the pancreas may be required. In addition, this entity is observed predominantly in younger female patients and is located in the body and/or tail of pancreas $(1,2)$, as observed in the current patient. The present case was further treated not only with intraoperative radiotherapy, but also postoperative chemotherapy for 20 months until recurrence was detected, and subsequently with chemoradiotherapy. The chemotherapy regimen in the present case was a combination chemotherapy with gemcitabine and S-1, one of the standard chemotherapeutic regimens for advanced pancreatic cancer (12).

The present case highlighted the management of MCN with anaplastic carcinoma of the pancreas in a Jehovah's Witness patient undergoing pancreatic surgery. Additional reports of this entity are required to optimize the standard management for this uncommon disease.

\section{References}

1. Suzuki Y, Atomi Y, Sugiyama M, Isaji S, Inui K, Kimura W, Sunamura M, Furukawa T, Yanagisawa A, Ariyama J, et al: Cystic neoplasm of the pancreas: A Japanes multi-institutional study of intraductal papillary mucinous tumor and mucinous cystic tumor. Pancreas 28: 241-246, 2004.

2. Sugiyama M, Suzuki Y, Abe N and Atomi Y: Surgical treatment of pancreatic cystic neoplasms. Nihon Shokakibyo Gakkai Zasshi 101: 865-871, 2004 (In Japanese).

3. Hakamada K, Miura T, Kimura A, Nara M, Toyoki Y, Narumi S and Sasak M: Anaplastic carcinoma associated with a mucinous cystic neoplasm of the pancreas during pregnancy: Report of a case and a review of the literature. World J Gastroenterol 14: 132-135, 2008.

4. Pan Z and Wang B: Anaplastic carcinoma of the pancreas associated with a mucinous cystic adenocarcinoma. A case report and review of the literature. JOP 8: 775-782, 2007.

5. Logan SE, Voet RL and Tompkins RK: The malignant potential of mucinous cysts of the pancreas. West J Med 136: 157-162, 1982.

6. Marinho A and Nogueira R, Schmitt F and Sobrinho-Simoes M: Pancreatic mucinous cystadenocarcinoma with a mural nodule of anaplastic carcinoma. Hispathology 26: 284-287, 1995.

7. Lane RB Jr and Sangüeza OP: Anaplastic carcinoma occurring in association with a mucinous cystic neoplasm of the pancreas. Arch Pathol Lab Med 121: 533-535, 1997.

8. Jeon YB, Yun S and Choi D: Transfusion free radical antegrade modular pancreaticoduodenectomy of metastatic neuroendocrine tumor of the pancreas in Jehovah's Witness patient. Ann Surg Treat Res 88: 106-110, 2015.

9. Konstantinidis IT, Allen PJ, D'Angelica MI, DeMatteo RP, Fischer ME, Grant F, Fong Y, Kingham TP and Jarnagin WR: Pancreas and liver resection in Jehovah's Witness patient: Feasible and safe. J Am Coll Surg 217: 1101-1107, 2013.

10. Japan Pancreas Society (eds): Classification of Pancreatic Carcinoma. 3rd edition. Kanehara \& Co., Ltd., Tokyo, 2011.

11. Piccini G, Pasculli A, D'Ambrosio E, Gurrado A, Lissidini G and Testini M: Retrospective comparison of traditional vs. Liga Sure impact dissection during pancreatoduodenectomy: How to save money by using an expensive device. Surg Technol Int 23: 88-93, 2013.

12. Li Y, Jiang Z, Zhang L and Liu G: Gemcitabine and S-1 combination therapy versus gemcitabine alone for locally advanced and metastatic pancreatic cancer: A meta-analysis of randomized controlled trials in Asia. J Chemother 27: 227-234, 2015. 